\title{
Controlled trial of mobilisation and manipulation for patients with low back pain in general practice
}

\author{
H SIMS-WILLIAMS, M I V JAYSON, S M S YOUNG, H BADDELEY, E COLLINS
}

British Medical fournal, 1978, 2, 1338-1340

\begin{abstract}
Summary and conclusions
Ninety-four patients with non-specific lumbar pain who were seen by their general practitioners took part in a double-blind controlled trial to compare mobilisation and manipulation with placebo physiotherapy. Immediately after treatment most patients showed improvements in the various features studied, but for several features improvement was more common in the treated group than the controls. At three months the condition of most patients was still improved but the differences between the two groups had largely disappeared. At one year the groups were identical. Prognostic presenting features were sought, but only a shorter history correlated with clinical improvement.

This study indicates the high rate of spontaneous resolution of low back pain. A course of mobilisation and manipulation may hasten improvement but does not affect the long-term prognosis.
\end{abstract}

\section{Introduction}

Back pain is among the commonest rheumatological complaints and is responsible for a substantial proportion of total morbidity and loss of work through illness. Objective data evaluating its treatment are few. This is due partly to the difficulties in diagnosing the precise cause of a patient's symptoms and in assessing progress and partly to lack of knowledge of the natural history of back-pain syndromes. We report here on a controlled trial of treatment of non-specific back pain based on a widely used form of physiotherapy-namely, mobilisation and manipulation as described by Maitland. ${ }^{1}$ In this first stage of a two-part trial we studied patients presenting with back pain to their general practitioners; in a subsequent study, to be reported separately, we assessed patients attending hospital rheumatological and orthopaedic clinics.

Department of Medicine, University of Bristol, Bristol
H SIMS-WILLIAMS, $\mathrm{MB}, \mathrm{CHB}$, research fellow

University of Manchester, Rheumatic Diseases Centre, Hope Hospital, Salford M6 8HD

M I V JAYSON, MD, FRCP, professor of rheumatology

Bristol Royal Infirmary, Bristol

S M S YOUNG, MCSP, physiotherapist

University of Queensland, Australia

H BADDELEY, DMRD, FRCR, professor of radiology

University of Bath, Bath

E COLLINS, BSC, statistician

\section{Patients and methods}

The patients were aged 20-65 years and had low back pain sufficien for their general practitioners to request radiographs of the lumbar spine from the open $x$-ray service organised by the Bristol hospitals. It was not thought worth while to sample every patient with back pain since many have trivial problems for which treatment is not indicated. Before entering the trial the patient had to agree to attend physiotherapy sessions for one month, and for subsequent assessments. Patients were excluded from the study for the following reasons: gross psychological disturbances; pregnancy; previous spinal surgery; inflammatory or other specific disorders of the spine, such as ankylosing spondylitis, Paget's disease, vertebral collapse; bladder or bowel disturbances; muscle wasting; other medical disorders that migh contraindicate the forms of treatment used; and if they were though by the physiotherapist to be unlikely to benefit from mobilisation. Patients with sensory abnormalities, loss of a reflex, and muscle weakness without wasting were included.

\section{CLINICAI. ASSESSMENTS}

Assessments were made by a physician without knowledge of which treatment was given. In obtaining the clinical history and at physical examination particular emphasis was placed on the spine. Flexion, extension, and lateral flexion of the lumbar spine and straight-leg raising were measured with a goniometer." : Radiographs of the lumbar spine were scored for changes in the intervertebral disc spaces and vertebral end-plates, the development of osteophytes, and apophyseal joint changes.

Repeat assessments were performed after the four-week course of treatment, two months later, and by postal questionnaire after one year. Patients who failed to reply to the questionnaire were visited at home. Subjective assessments of pain, return to normal activity, opinions on the value of treatment, need for further treatment, and objective measurements of spinal mobility and straight-leg raising were recorded.

\section{TREATMENT}

The patients were randomly allocated to either active or placebo (control) physiotherapy. The active treatment, based on the mobilisation and manipulation of the spine described by Maitland' and widely used in physiotherapy departments, was given by a physiotherapis experienced in these techniques (SMSY). This treatment programme entails detailed and repeated assessments of the spine to localise the levels responsible for the symptoms. Treatments are directed at these levels, although inevitably they also affect adjacent levels. The programme is modified according to the patient's progress. Passive mobilisation comprises oscillatory movements, including rotation techniques and accessory movements which can be given in the posteroanterior or transverse directions. 'Traction-either intermitten or continuous-is used alone or combined with mobilisation and is directed at the pain-producing levels. Less commonly, manipulation techniques are used-that is, small-amplitude, high-velocity movements going beyond the normal active ranges of motion. Abdominal and general active spinal exercises are given as necessary, and occasionally local heat is applied.

Placebo physiotherapy was given by the same physiotherapist with comparable degrees of enthusiasm. Microwave radiation at the lowest possible setting was given to the lumbar spine for 15 minutes with the patient prone unless this caused pain. 
Treatments were given daily for one week and then thrice weekly for three weeks but could be stopped earlier if the symptoms were relieved or if they advanced so that further investigation or treatment was indicated.

\section{Results and comment}

One hundred and eight patients were suitable for the study but 14 failed to attend for assessment or treatment. Thus 94 entered the trial and were randomly allocated into groups receiving active and control treatments; these groups were basically homogeneous (tables A and $\left.\mathrm{B}^{*}\right)$.

\section{ONE-MONTH ASSESSMENT}

Eighty-seven patients attended for follow-up at one month, immediately after the course of physiotherapy. Reduced pain was evident in most cases. This was more common in patients who had received active treatment than the controls, but the difference was only of borderline significance (table C). Most patients, but significantly more of those in the active-treatment group than the controls, could perform at least light work (table D). Most patients thought that their treatment, whether active or placebo, was helpful. This opinion was more common among those who had received active treatment but the difference was only just significant (table E).

Measurements of the ranges of spinal motion and straight-leg raising at one month were compared with those made initially (table F). No change in flexion had occurred in the active-treatment group but there was a significant decrease in the controls. Both groups showed highly significant improvements in extension. Lateral flexion to the right or left remained the same in both groups. Straight-leg raising had improved significantly on both sides in those who had received active treatment but not in the controls.

Overall, the condition of all the patients seemed to have improved with physiotherapy, but the improvement was more noticeable in the group who had received active treatment.

\section{THREE-MONTH ASSESSMENT}

We assessed 83 patients three months after they entered the trialthat is, two months after the end of treatment. Symptoms were still improved in most patients, although improvement was less common than it had been at one month and did not differ between the two groups (table G). Most patients could perform some work, the proportions in the two groups being similar (table $\mathrm{H}$ ).

Most patients still felt that their treatment had been beneficial, although fewer thought this than had done so at the one-month analysis. This view was still more commonly held by those who had received active treatment, but the difference only bordered on significance (table I).

Objective measurements (table J) showed no change in spinal flexion in those who had received active treatment, but the significant deterioration in the controls was maintained. Both groups still showed significant improvements in extension. No change had occurred in right lateral flexion and right or left straight-leg raising, but left lateral flexion had improved in the active-treatment group.

Thus most patients' conditions were still improved at three months but the proportion was smaller than it had been immediately after the end of treatment. The patients who received mobilisation and manipulation showed slightly greater improvements than the controls but the differences seen at one month had largely disappeared.

\section{ONE-YEAR ASSESSMENT}

Information was obtained by postal questionnaire or personal visit from 90 of the 94 patients. Most thought that their backs were better than when they had first been seen, but there was no difference between the two groups (table K). Physical activity was still limited in many patients, the proportions in both groups being similar (table L). Patients were asked to state whether they thought their treatment had conferred temporary or permanent benefit: answers were similar in both groups (table $M$ ). Many patients had received further treatment to their spines after the trial period but the proportions were similar in both groups (table N). Further analysis of the data to see whether *Tables A-Q may be obtained from the authors. additional treatment affected prognosis yielded no information of value.

Thus the condition of about two-thirds of the patients was significantly better at one year than at the initial assessment. Many patients still had recurrent spinal problems, and there were no differences between those who had received active and placebo physiotherapy.

\section{PROGNOSTIC FACTORS}

Presenting features were compared between the 30 patients who did best and worst immediately after treatment. No correlation was found between prognosis and age, sex, marital status, physical activity, sudden or gradual mode of onset, precipitating cause, root pain aggravated by raising intrathoracic pressure, radiation of pain, straightleg raising, radiographic changes in the lumbar spine, and even whether they received active or placebo treatment (table O). Patients with pain duration of less than one month more frequently improved than those with a history of greater than a month (table P).

Virtually no differences were found at one year between these two groups of 30 patients, indicating that the long-term prognosis was no better for those who did well initially than for those who did poorly (table Q)

\section{Discussion}

Although the cause of back pain may be identified in some patients, commonly the diagnosis is in doubt, in which case patients are said to have "non-specific back pain." Many different conditions are included within this group, so that it may be difficult to assess the value of any form of treatment. What happens in the spine during manipulation and how it relieves pain is disputed. Mathews and Yates ${ }^{4}$ suggested that posterior disc bulges could be reduced by rotational manipulation tightening the posterior longitudinal ligament and exerting a centripetal pressure on the protrusion. It is difficult, however, to see how such a mechanism could do more than temporarily relieve symptoms. Other possibilities include freeing adhesions around a prolapse and mechanically stimulating large nerve fibres inhibiting transmission of nociceptive impulses through small-diameter nerve fibres.

Doran and Newell' compared manipulation, physiotherapy excluding manipulation, corset, and analgesics in patients with back pain. They failed to identify any important differences, although some patients responded well and quickly to manipulation. Different forms of manipulation were used at each of the six contributing centres, however, and the trial was subject to considerable criticism. Evans et $a^{6}{ }^{6}$ conducted a cross-over tria comparing three rotational manipulations at weekly intervals with analgesics alone. They found improvements in spinal flexion and pain scores related to the treatment period. The study's design, however, meant that for some patients the control period was the first form of treatment but for others it followed manipulation, making interpretation of the results difficult. Glover et al' compared manipulation with detuned short-wave diathermy in people in a factory who had back pain. Many who were manipulated obtained immediate relief, but after a few days there was no difference between the two groups. BergquistUlmann and Larsson ${ }^{*}$ compared spinal mobilisation, instruction on the care of the back, and placebo physiotherapy in people with back pain. Mobilisation and instruction were better than placebo for certain parameters.

We used Maitland's form of mobilisation and manipulation. ${ }^{1}$ Many other forms exist, and all may be practised in different ways by different practitioners. Caution is indicated in drawing broad conclusions on the values of mobilisation and manipulation from any study.

We emphasise that this study was carried out only on patients seen by general practitioners. Patients attending hospital rheumatological and orthopaedic clinics represent a different group of sufferers, since they have more-severe symptoms that are sufficient to warrant a specialist opinion and have persisted 
long enough for the general practitioner to have referred them to hospital and for them to have been seen in the clinic. As most improvements occurred in patients with a short history of symptoms hospital patients would probably not show such a high rate of improvement.

The results suggest that most sufferers from back pain obtain relief without any specific treatment, and that mobilisation and manipulation may hasten this improvement but make no difference to the long-term prognosis. Moreover, although at the time more patients found the active treatment helpful, in retrospect they failed to distinguish active from placebo physiotherapy. For some patients with back pain prolonged or recurrent courses of mobilisation and manipulation would perhaps provide long-term relief, but an appropriate trial is needed. We were unable to identify any factor other than a short history that might indicate a good prognosis. This is disappointing, as prognostic markers would be helpful not only in managing the individual patient but also in leading to a better understanding of the problem.
This study was performed with the aid of a grant from the DHSS, to whom we are most grateful.

Copies of the tables may be obtained from Professor M I V Jayson, University of Manchester, Rheumatic Diseases Centre, Hope Hospital, Salford M6.

\section{References}

${ }^{1}$ Maitland, G D, Vertebral Manipulation, 4th edn. London, Butterworths, 1977.

2 Locbl, W Y, Annals of Physical Medicine, 1967, 9, 103.

${ }^{3}$ Reynolds, P M G, Rheumatology and Rehabilitation, 1975, 14, 180.

${ }^{4}$ Mathews, J A, and Yates, D A H, British Medical fournal, 1969, 3, 696

5 Doran, D M L, and Newell, D J, British Medical fournal, 1975, 3, 161.

${ }^{6}$ Evans, D P, et al, Rheumatology and Rehabilitation, 1978, 17, 46.

Glover, J R, Morris, J G, and Khosla, T, British fournal of Industrial Medicine, 1974, 31, 59.

* Bergquist-Ulmann, M, and Larsson, U, Acta Orthopaedica Scandinavica, 1977, suppl No 170 .

(Accepted 15 September 1978)

\section{SHORT REPORTS}

\section{Greater auricular nerve in diagnosis of leprosy}

In its early stages leprosy is usually diagnosed on clinical grounds, ${ }^{1}$ and great emphasis is laid on finding thickened nerves. ${ }^{2}$ Indeed, in endemic areas thickened nerves are sometimes accepted as diagnostic of the disease. ${ }^{3}$ An exact definition of what constitutes a thickened nerve is therefore of considerable importance. The greater auricular nerve is particularly valuable in this respect because as well as being often affected in leprosy it is said to be rarely palpable normally. ${ }^{1}$

\section{Subjects, methods, and results}

Three hundred and thirty-seven 17-year-old Nepali recruits to Britain's Brigade of Gurkhas were examined to find the frequency with which one or both of their greater auricular nerves were either visible or palpable. Their mean $( \pm S D$ ) height was $124 \pm 8 \mathrm{~cm}$ and weight $64 \cdot 15 \pm 1 \cdot 7 \mathrm{~kg}$. They were examined with the head laterally rotated and the neck extended $20^{\circ}$. The nerve, when detectable, was then parallel with and $1.5 \mathrm{~cm}$ lateral to the external jugular vein. The whole body surface was examined in good light for other stigmata of leprosy, including hypopigmented patches and thickening of the ulnar, lateral popliteal, and other cutaneous nerves.

The greater auricular nerve was visible or palpable on one or both sides in $212(63 \%)$ subjects and ranged in diameter from 2 to $4 \mathrm{~mm}$. In 21 , one or both ulnar nerves were considered thickened. None had detectable lateral popliteal nerves, and no palpable nerves were tender. Four had hypopigmented patches, not obviously due to tinea versicolor, but none of these was hypoaesthetic.

\section{Comment}

Most studies of the greater auricular nerves in endemic leprosy areas have been concerned with the prevalence of abnormally thickened nerves in leprosy in the absence of other signs, and this has been about $1.5^{\circ}, 0^{45}$ Despite a widely held opinion that the normal nerve is seldom palpable, ${ }^{1}$ I was unable to find a study similar to this one, the purpose of which was mere detection of the nerve, thickened or not. The population studied perhaps favoured detection in that they were young, fit, and slim and had well developed neck muscles (perhaps a result of carrying heavy loads on "dokos," or wicker baskets, supported by a head band). Nevertheless, such a high rate of detection induces a note of caution in assessing the usefulness of this clinical sign. A further problem in distinguishing normal from leprous nerves has been a bulbous swelling, or perhaps kinking, of the nerve commonly present immediately as it emerges from behind the sternomastoid muscle to assume a subcutaneous position.

Leprosy is rare among soldiers of the Brigade of Gurkhas. There has been no new case since 1970 . We may reasonably conclude therefore that the greater auricular nerve is often detectable in normal individuals from an endemic area and that its usefulness in the diagnosis of leprosy is thereby diminished.

${ }^{1}$ Bryceson, A, Medicine, 1978, 3, 171.

2 Murdock, J R, International fournal of Leprosy, 1949, 17, 1.

3 Lowe, J, Leprosy Notes, 1929, 5, 7.

4 Gehr, E, and Stolze, E, Documenta de Medicina Geographica et Tropica, 1956, 8, 351.

${ }^{5}$ Leiker, D L, and Sloan, N R, International fournal of Leprosy, 1954, 22, 431 .

(Accepted 8 September 1978)

British Military Hospital, Dharan, Nepal

PETER LYNCH, MRCP, major, RAMC (present address: Royal Army Medical College, Millbank, London SW 1)

\section{Behçet's disease and splenomegaly}

Behçet's disease has been defined as recurrent oral and genital ulceration with iridocylitis. ${ }^{1}$ It appears to be a systemic disease affecting skin and vascular, locomotor, central nervous, and gastrointestinal systems." Two cases with splenomegaly are described. We did not find a cause for the enlarged spleen and we assumed that it was part of the disease spectrum.

\section{Case reports}

(1) A 29-year-old man presented in November 1976 with a five-year history of oral and scrotal ulcers, intermittent fever, and acne. Previous history included epilepsy diagnosed in 1969 and treated with phenobarbitone and phenytoin. On examination, he had multiple oral and scrotal ulcers, acneiform lesions of the face, and clubbing of fingers and toes. The spleen was palpable $15 \mathrm{~cm}$ below the costal margin. The liver was firm and enlarged $10 \mathrm{~cm}$ below the costal margin. The haemoglobin was $11.6 \mathrm{~g} / \mathrm{dl}$, the red cells showed anisopoikilocytosis, and the white blood count was $5 \cdot 8 \cdot 10^{9}$ $\left(5800 \mathrm{~mm}^{3}\right)$ with a relative lymphocytosis. The ESR varied between 45 and $105 \mathrm{~mm}$ Westergren in the first hour. Liver function tests and prothrombin time were normal. Serum protein electrophoresis showed diffuse hyperglobulinaemia. The Paul-Bunnell toxoplasma and brucella tests were normal. Tests for antinuclear factor (ANF) were positive on two occasions and negative on three other occasions. Phenytoin was thought to be responsible for the positives. Tests for anti-DNA antibodies were negative. HLA types were A1, A10, B5, and B12. Growth hormone was normal. Oesophago- 